“ C 2019 IEEE. Personal use of this material is permitted. Permission from IEEE must be obtained for all other uses, in any current or future media, including

reprinting/republishing this material for advertising or promotional purposes, creating new collective works, for resale or redistribution to servers or lists, or reuse of any copyrighted component of this work in other works." 


\title{
3-D Printed Strain Sensor for Structural Health Monitoring
}

\author{
Nuwan Munasinghe*, Matthew Woods*, Lewis Miles ${ }^{\dagger}$, Gavin Paul* \\ ${ }^{*}$ Centre for Autonomous Systems, University of Technology Sydney (UTS), Sydney, Australia \\ Email: \{Nuwan.Munasinghe,Matthew.J.Woods\}@student.uts.edu.au \\ Email: Gavin.Paul-1@uts.edu.au \\ ${ }^{\dagger}$ UTS Rapido, University of Technology Sydney (UTS), Sydney, Australia \\ Email: Lewis.Miles@uts.edu.au
}

\begin{abstract}
Additive manufacturing, or 3D printing, is evolving from a technology that can only aid rapid prototyping, to one that can be used to directly manufacture large-scale, real-world equipment. Gravity Separation Spirals (GSS) are vital to the mining industry for separating mineral-rich slurry into its different density components. In order to overcome inherent drawbacks of the traditional mould base manufacturing methods, including significant tooling costs, limited customisation and worker exposure to hazardous materials, a 3D printer is under development to directly print spirals. By embedding small Internet of Things (IoT) sensors inside the GSS, it is possible to remotely determine the operation conditions, predict faults, and use collected data to optimise production output. This work presents a 3D printed strain sensor, which can be directly printed into the GSS. This approach uses a carbon-based conductive filament to print a strain gauge on top of a Polylactic Acid (PLA) base material. Printed sensors have been tested using an Instron E10000 testing machine with an optical extensometer to improve accuracy. Testing was conducted by both loading and unloading conditions to understand the effect of hysteresis. Test results show a near-linear relationship between strain and measured resistance, and show a $6.05 \%$ increase in resistance after the test, which indicates minor hysteresis. Moreover, the impact of viscoelastic behaviour is identified, where the resistance response lags the strain. Results from both conductive and non-conductive material show the impact of the conductive carbon upon the tensile strength, which will help to inform future decisions about sensor placement.
\end{abstract}

Index Terms - strain gauge, additive manufacturing, IoT, 3Dprinted sensor, infrastructure monitoring

\section{INTRODUCTION}

Additive manufacturing, also known as $3 \mathrm{D}$ printing is the process of printing a physical object from a computer designed model. During recent years, additive manufacturing has transformed into a viable end-product manufacturing technique adopted by various industries [1] such as in medicine, aerospace, and construction [2]-[6]. During its operational lifetime, these $3 \mathrm{D}$ printed objects can be exposed to various environmental conditions like vibration, humidity, general wear, and temperature variations. Therefore, it is important to evaluate the performance under expected condition so as to reduce unexpected failures and plan maintenance activities so they do not disrupt operations. Ideally, these health monitoring

This work is being supported by the University of Technology Sydney (UTS), the Innovative Manufacturing Cooperative Research Centre (IMCRC) and Mineral Technologies. systems should be low cost and fulfil requirements like the ability to perform a continuous assessment, an insensitivity to ambient loading and noise, and be robust to changes in environmental conditions [7].

A research project is currently underway to develop a 3D printer to print Gravity Separation Spirals (GSS), which can avoid the drawbacks inherent to the traditional GSS manufacturing process shown in Fig. 11. These GSS are used in the mineral industry to separate minerals from the slurry. The slurry is poured into the top and then the spiral slope naturally helps separate the slurry due to the different particle density. The size of these GSS vary depending on the application (approximately $2.5-4 \mathrm{~m}$ ) and ideally the slope of the spiral profile can be modified slightly for every single customer, depending upon the composition of their slurry and their desired outcomes. Such mass customisation is uneconomical with the traditional mould-based manufacturing process, but it is straightforward using additive manufacturing. The team has already developed a $1 / 3$ scale prototype $3 \mathrm{D}$ printer. One objective of this project is to embed sensors into the 3D-printed GSS to remotely monitor the operational conditions. Currently, the spirals are combined into large banks as shown in Fig. 1p, and operate in remote mining locations all around the world, such as in South Africa, USA, India, Brazil, and Australia. This project aims to avoid or eliminate the time-consuming and costly need for technicians to travel around the world to troubleshoot issues. Additionally, through a detailed history of usage, which embedded sensors can provide, fault diagnosis can be significantly improved. Two key advantages of 3D printed sensors, instead of embedding conventional sensors, is that they are low-cost and can be integrated into the large build volume of the structural material without compromising the mechanical integrity of the object [8]. A 3D printed wear profiling sensor has previously been developed and tested for this application [9]. This current paper presents the recent development of a new strain sensor that can be integrated into the 3D-printed GSS.

Measuring strain has many advantages in this application for several reasons. One reason is to confirm that operators are following the provided guidelines and do not put unnecessary loads on the GSS. Under excessive loads a GSS can deform permanently, which may result in the need for them to be 


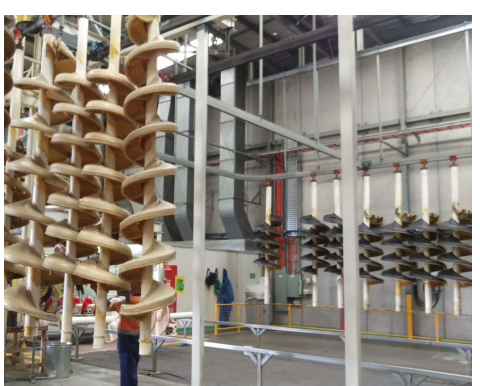

(a)

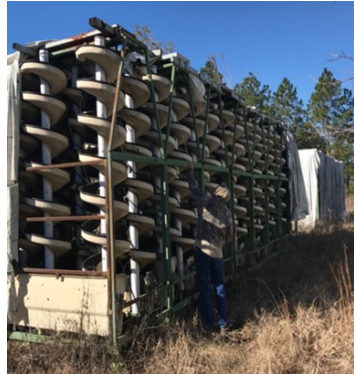

(b)
Fig. 1: a) GSS manufacturing factory. b) Bank of GSS installed in the field.

replaced, costing both time and money. Monitoring strain remotely will help to identify these kinds of scenarios and help to alert operators to imminent faults, or advise them on how best to follow the guidelines that prevent damage. Another reason is to measure and understand the long-term creep these spirals are subject to, and predict and advise an operator to replace them when necessary. The production output of the GSS is highly dependent on the shape and profile of the spiral [10]. In time, the material will deform slowly under load (i.e. creep), and this will likely reduce the production output. Monitoring this will provide data that can be used to predict future maintenance or advise if a replacement is required. By understanding the long-term creep behaviour of a GSS, it may be possible to modify the design to mitigate the previously observed and undesirable creep behaviour in the future.

It is vital to measure internal strains, associated stresses or vibrations in structural members to gather information about the health and the performance of a system [8], [11], [12]. Strain gauge characteristics vary greatly to meet the requirements of many applications and industries including stretchable strain sensors used in measuring elbow flexing, finger movement tracking for virtual-reality, assessment of postures in textiles and measuring bending and contact detection in soft actuators [13]-[17].

Primarily, strain gauges are based on the deformation of conductive material when they are subjected to strain. These sensors are composed of deformable conductive material attached or encapsulated in a base material. These deformations can be measured by changes in capacitance or resistance. Capacitance-based sensors most commonly consist of a dielectric layer sandwiched between conductive layers. Layer thickness changes due to applied loads will result in a capacitance change of the sensor [18]. Frutiger et al. [19] developed a capacitance-based wearable strain sensor using multicore-shell fibre printing. Stretching the sensor will reduce the distance between internal layers increasing its capacitance value. On the other hand, resistance-based strain gauges measure changes in resistance due to strain induced geometry changes [20]. A consideration for this application is that the dielectric constants of capacitance-based sensors can be affected by changes in moisture content. As the sensors will be embedded in spirals that work in direct contact with a wet slurry, a resistance based sensor is more suitable for this application.

Researches have used a variety of 3D printing technologies to print strain gauges. A mould-based manufacturing method has been developed by Nag et al. [21] for low-force applications, where a 3D printed mould is used to cast graphite powder for the sensor traces. Extrusion-Based approaches have been used to extrude metallic paste or viscoelastic ink to print strain gauges [13], [22]. Fused Deposition Modeling (FDM) based 3D printing was used by Michael et al. [11] to understand the relationship between strain and resistance for carbon black filled Acrylonitrile Butadiene Styrene (ABS). Since the 3D printer under development for the overall project is based on FDM technology, this research was carried out to develop a suitable FDM-based approach to print strain gauges for the printed spirals.

The selection of a conductive material for the strain sensor is highly dependent on the nature of the application and the electromechanical properties of the material. There are a variety of conductive 3D printable materials available, which are either metallic or non-metallic. In liquid metal based strain gauges, resistance is directly related to the deformation of the channels holding the liquid. These sensors are suitable for highly stretchable applications. Commonly used liquid metals include mercury and gallium [14], [23]. Various allotropes of carbon have been used for different sensing applications. Graphite has been used due to its high electrical conductivity, corrosion resistance, and porosity [21]. But on the other hand, carbon-based solutions suffer from hysteresis, which lead to the changes in electrical resistivity [19]. A carbon-based filament was selected as the sensor material in this research for its high availability, low-cost, and corrosion resistance. Additionally, a wear sensor was developed for the same application using the same material [9], which should help to reduce the overall production complexity.

The objective of this research is to design a strain gauge configuration which is resilient to environmental changes, such as temperature variations and can compensate for transverse strains. There are various configurations of strain gauges like a quarter bridge, half-bridge and full-bridge, which have different measurement types, compensation and sensitivity [24]. Finally, the sensors will be printed in to spirals and will be connected to a small IoT device which will measure strain and send data to a remote location. At this stage of the research, authors are focused on understanding the behaviour and characteristics of 3D printed material under stress. Therefore, this paper presents the test results of a single 3D printed strain gauge. The rest of the paper is organised as follows: Section II describes the theory of strain gauges and material adhesion properties; Section IIII presents the specimen printing process and conducted experimental results, Section IV provides the discussion of results, and finally, Section $\mathrm{V}$ presents the conclusions and future research work. 


\section{Methodology}

\section{A. Principle of Strain Gauges}

Strain is the ratio of elongation over its original length due to an applied force. There are four types of strain: axial, bending, torsional and shear [24]. This research is focused on axial strain. In resistance-based strain gauges, the resistance varies proportionally to the strain on the device. Fig. 2 shows the basic components of a strain gauge. A traditional strain gauge consists of thin conductive metallic foil or wire in a grid pattern to maximise the amount of axial strain that traces are subject to. In this research, 3D printed conductive carbon traces are used as a replacement to metallic foil or wire. These traces are printed directly onto a base material, which transfers the strain to the traces.

The resistance of the material according to the subjected deformation is given by (1), where $R$ is the resistance, $\rho$ is the resistivity, $l$ is the length, and $A$ is the cross-sectional area. The strain due to the applied load will reduce $A$ and increase $l$, which will increase the resistance in terminals $A$ and $B$ in Fig. 2. The ratio of the fractional change in resistance, $\Delta R / R$ is proportional to strain, as shown in (2). The gauge factor, $G F$ defines the gauge sensitivity to the strain $\varepsilon$.

$$
\begin{gathered}
R=\rho l / A \\
\Delta R / R=G F \times \varepsilon
\end{gathered}
$$

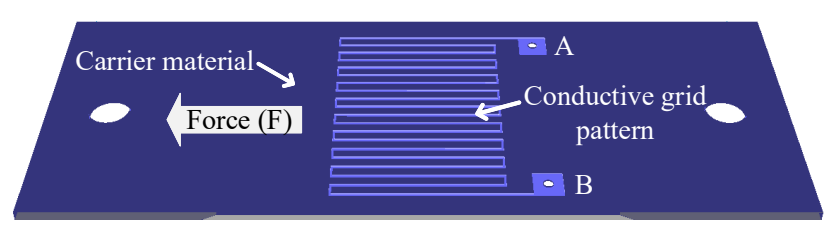

Fig. 2: Components of a strain gauge.

\section{B. Material Adhesion Properties}

Thermoplastics can be joined by heating the interface surfaces to their melting point, then allowing polymer chains to form inter-molecular bonds [25]. The strength of the interface joint is controlled by variables including material melting temperatures, chemical compatibility, temperature, joining pressure, time and material surface energy [26], [27]. It has been shown that multi-material prints have reduced strength due to lower layer adhesion strength between different materials [28]. Thus, in producing a multi-material print with maximum strength, the chemical compatibility and melting temperature of the materials is a key consideration.

\section{Sensor Calibration}

Calibration of strain gauges is performed by applying known loads to the gauge and measuring the resulting output. When strain gauges are attached to any equipment, the ideal calibration method is to load the equipment with a known stress distribution and monitor the output [29]. To calibrate the printed strain gauge, a tensile testing machine can be used. While applying known strain values to the gauge, the electrical resistances for a given strain setting can be measured in order to determine the gauge factor. Once the gauge factor is determined, the sensor is said to be calibrated and future measurements of the resistance can be used to determine the strain.

\section{EXPERIMENTAL RESULTS}

\section{A. Testing Summary}

To show the validity of the 3D printed strain gauge and for sensor calibration, an Instron E10000 tensile testing machine was used. Dog bone shaped printed samples of different materials and with various raster angles were tested to understand the material properties. 3D printed strain gauges were tensile tested to investigate the relationship between resistance and elongation.

\section{B. Materials}

For conductive traces, Proto-Pasta conductive Polylactic acid (PLA) was chosen. This material is a composite comprised of Natureworks 4043D PLA mixed with conductive carbon black. Its resistivity is rated for $15 \Omega . \mathrm{cm}$ before printing and after printing, $30 \Omega . \mathrm{cm}$ along the $\mathrm{X}-\mathrm{Y}$ plane and 115 $\Omega . c m$ in the $\mathrm{Z}$ axis. Initially, specimens were printed with a base material of Thermoplastic Polyurethane; however, poor layer adhesion resulted in the traces detaching. To improve the layer adhesion between the base and conductive traces, non-conductive PLA was selected for its comparable material properties and printing parameters.

\section{Printing Process}

The specimens were printed on a Prusa i3 MK3 with the Multi-Material Unit 2.0. The printer is a popular open source FDM-based printer, which enables the printing of up to five materials. The printer was used as there are a wide variety of cheap, accessible materials that can be printed.

Table I shows the print parameters that were used to print the specimens.

TABLE I: Print Parameters

\begin{tabular}{|l|l|}
\hline Print Parameter & Value \\
\hline Nozzle diameter & $0.4 \mathrm{~mm}$ \\
Bed temperature & $65^{\circ} \mathrm{C}$ \\
Nozzle temperature for PLA & $210^{\circ} \mathrm{C}$ \\
Nozzle temperature for conductive PLA & $240^{\circ} \mathrm{C}$ \\
Printing speed PLA & $60 \mathrm{~mm} / \mathrm{s}$ \\
Printing speed conductive PLA & $25 \mathrm{~mm} / \mathrm{s}$ \\
Purge volume & $700 \mathrm{~mm}$ \\
\hline
\end{tabular}

All specimens were printed with $100 \%$ infill and a layer height of $0.15 \mathrm{~mm}$. Two types of dog bones were printed in each material with a 45-degree raster angle and $100 \%$ axial layer direction. The dog bone with $100 \%$ axial layers results in all printed strands oriented parallel to the tensile load. The 45-degree raster is offset from the tensile load by 45 -degrees with each alternating layer oriented by -45 -degrees. The strain gauge base was printed with a 45-degree raster angle, and the traces were printed with a single strand per layer. 


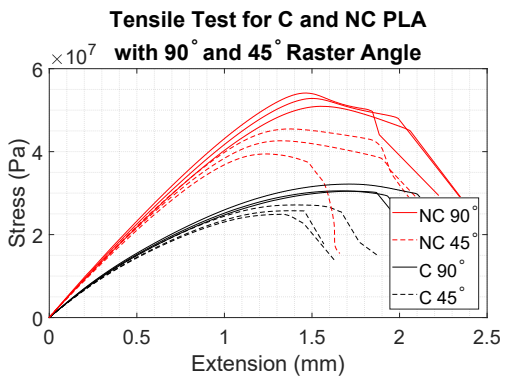

(a)

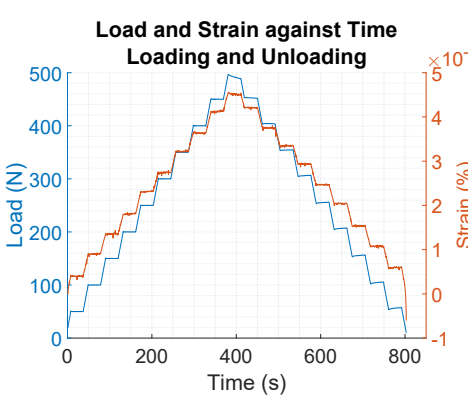

(b)

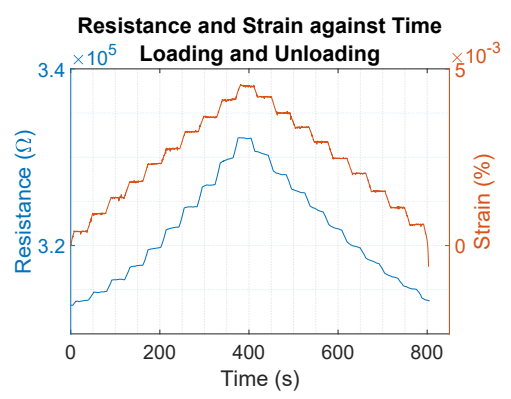

(c)

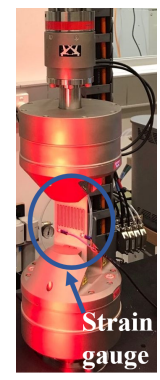

(d)

Fig. 3: (a) Standard tensile tests for conductive (C) and non-conductive (NC) PLA with $90^{\circ}$ and $45^{\circ}$ raster angles. (b) Load and strain data for loading and unloading tests. (c) Resistance and strain data for loading and unloading tests. (d) Testing strain gauge using Instron E10000 machine.

\section{Specimen Details}

The dog bone dimensions were followed from the ASTM D638, a standard test method for tensile properties of plastics [30]. The printed dog bones are shown in Fig 4a As a preliminary test, the strain gauge dimensions were determined by the printability and allowable specimen dimensions of the Instron E10000 tensile testing machine. To improve layer adhesion between the two materials, the conductive traces were embedded into the natural PLA, and the printing speed was reduced. To increase the distribution of the load across the specimen, the thickness of the base material was increased at the gripping points. The printed strain gauge is shown in Fig $4 \mathrm{~b}$. Table II presents the key dimensions of the specimens.

\section{TABLE II: Strain Gauge Dimensions}

\begin{tabular}{|l|l|}
\hline Dimension & Value (mm) \\
\hline PLA base depth & 0.8 \\
Trace depth & 0.4 \\
Trace width & 0.4 \\
Individual trace length & 40 \\
Total trace length & 869 \\
Trace offset distance & 3 \\
Length of strain surface & 90 \\
Width of strain surface & 69.5 \\
Tab thickness & 2.4 \\
\hline
\end{tabular}

\section{E. Testing}

1) Standard Tensile Testing: Tensile tests of the $100 \%$ axial strand layer dog bones were conducted to understand the characteristics of conductive and non-conductive PLA materials. The three specimens were tested in the Instron E10000 tensile testing machine at a constant elongation rate of $0.5 \mathrm{~mm} / \mathrm{min}$ until failure. To understand the effect of raster angles on the specimen strength, the 45-degree raster angle dog bones were tested using the same methodology.

2) Strain Gauge Testing: To determine the elastic stress range of the strain gauges, destructive tensile tests were conducted to identify the load at the point of plastic deformation. Strain gauge specimens were tested at an elongation rate of $0.25 \mathrm{~mm} / \mathrm{min}$ until failure. To determine the relationship

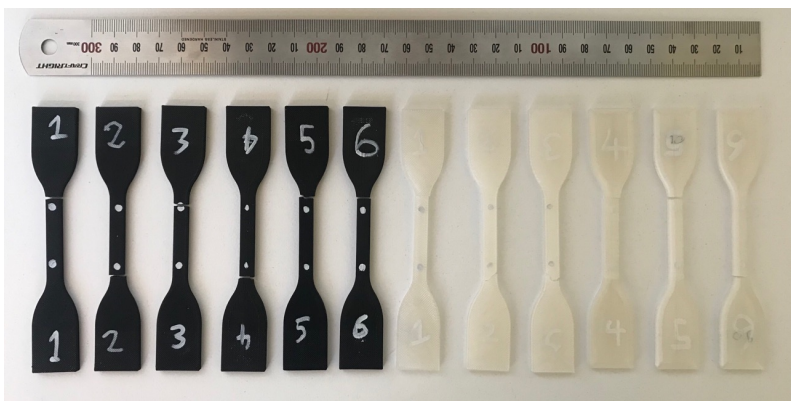

(a)

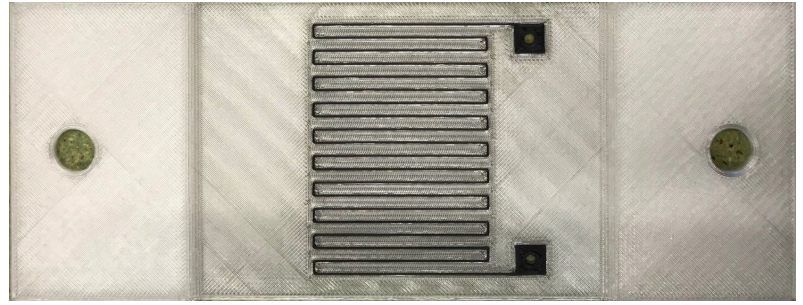

(b)

Fig. 4: a) ASTM D638 standard dog bones. b) 3D printed strain gauge.

between specimen strain and the printed trace resistance, the strain gauge was tensile tested within its elastic region. The specimens were loaded and unloaded in a staggered manner, with a constant load being held at increments of $50 \mathrm{~N}$ for 30 seconds while strain, load and resistance were recorded. The specimen being held at a constant load allowed for the observation of hysteresis due to creep or viscoelastic material effects.

\section{F. Results}

The standard dog bone testing stress-strain results can be seen in Fig 3a It can be observed that the conductive PLA had a lower Ultimate Tensile Strength (UTS) compared to PLA, with conductive PLA having a UTS of $30.45 \mathrm{MPa}$ and PLA having $53.69 \mathrm{MPa}$ for $100 \%$ axial strand specimens.

The 45-degree raster angle specimens showed a decrease in strength compared to the $100 \%$ axial strand specimens, with 
a reduction in the UTS of $15.6 \%(4.75 \mathrm{MPa})$ for conductive PLA and $19.11 \%$ (10.07 MPa) for PLA.

The destructive strain gauge testing shows a plastic deformation after a $0.6 \mathrm{kN}$ load and a UTS of $1.3 \mathrm{kN}$. Fig $3 \mathrm{~b}$ shows the load and strain against time for the strain gauge testing. It can be seen that under the constant loads there is no significant increase in strain due to plastic deformation, indicating the 500 $\mathrm{N}$ load is within the elastic range of the specimen.

Resistance and strain data for the strain gauge loading and unloading test is shown in Fig $3 \mathrm{c}$. The results show that there is a nearly linear relationship between elongation and resistance for the $3 \mathrm{D}$ printed strain gauge. There is a small level of hysteresis observed in the resistance with a $6.05 \%$ increase in resistance between the starting and finishing resistance of the test. During the unloading of the specimen, a lagging of resistance has been observed relative to the strain measurement. This is believed to be due to the viscoelastic behaviour of the PLA.

\section{Discussion}

The main objective of this research is to $3 \mathrm{D}$ print and characterise a strain gauge that can be used to measure long term strain or creep in 3D printed GSS.

Initial tensile tests have been conducted to understand the difference between the 3 -D printed conductive and nonconductive PLA. Tests indicated that there is an approximately $73 \%$ increase in UTS for the non-conductive filament. By changing the raster angle in the 3D-printed specimen, it has been observed that the raster angle can affect the tensile strength of the material. This understanding of material properties will help to improve the design of the strain gauge, to make optimisation decisions on the sensor placement, and to predict its effect on the structural integrity of the spiral.

By studying the relationship between the resistance and strain, it is clear that there is a near linear relationship between strain and resistance. This result indicates that the presented FDM 3D-printed strain gauge can be used to measure strain. An important observation of this research is the viscoelastic behaviour of the material and understanding its impact on the resistance change in relation to the strain. It was observed, due to the viscoelastic behaviour of the PLA, resistance values lag the change in strain. Even though this lagging resistance behaviour might not be suitable to measure strain changes occurring rapidly, it is not an issue in this specific application since strain due to creep will occur over a long period of time. It was also notable that hysteresis, due to network restructuring carbon particles in the conductive material, has not been observed. This indicates that no permanent damage was done to the traces.

One known limitation from this research is the material properties of PLA. PLA has a relatively low glass transition temperature of around $60^{\circ} \mathrm{C}$. If utilising darker coloured material this temperature could easily be reached in direct sunlight on a hot day resulting in deformation of the sensor. Moreover, since PLA is a biodegradable plastic, it absorbs water, which could alter the resistance of the print specimen as well as degrading it over time.
Calibration of the sensors on the spiral can be done by applying known loads to the spiral and determining the resistance and strain relationship. By conducting the calibration of sensors multiple times, the precision of the sensor can be determined. The embedded printed strain sensor will be integrated as an IoT device to enable the transmission of the data back to a central monitoring location.

\section{CONCLUSION}

This paper has presented a 3D printed strain sensor, which can be directly printed into large-scale, real-world equipment, such as gravity separation spirals, to remotely measure structural creep. Tests conducted with an Instron E10000 machine proved that there is a near linear relationship between resistance and strain with an increase of $6.05 \%$ between the initial and final resistances. Moreover, these tests helped to understand the impact of the viscoelastic properties on a printed strain gauge. Additionally, standard tensile tests were conducted with both conductive and non-conductive PLA with different raster angles to understand the material properties, and to calibrate the sensor.

Future research will focus on understanding the viscoelastic behaviour and its impact on strain measurement. Additional research is required to understand the long term suitability of sensor materials for measuring creep. Furthermore, different strain sensor configurations will be developed to incorporate temperature and transverse stress compensation.

\section{ACKNOWLEDGMENT}

This research is supported by the University of Technology Sydney, the Innovative Manufacturing Cooperative Research Centre (IMCRC) and Mineral Technologies. The authors would also like to thank Chris Chapman for helping to conduct the experiments on the Instron testing machine, and to Thomas Romeijn for his insights about plastic deformation behaviour.

\section{REFERENCES}

[1] K. V. Wong and A. Hernandez, "A Review of Additive Manufacturing," ISRN Mechanical Engineering, vol. 2012, no. 208760, p. 10, 2012.

[2] G. Z. Cheng, E. Folch, A. Wilson, R. Brik, N. Garcia, R. S. J. Estepar, J. O. Onieva, S. Gangadharan, and A. Majid, "3D Printing and Personalized Airway Stents," Pulmonary Therapy, vol. 3, no. 1, pp. 59-66, 2017.

[3] Y. Tadjdeh, "3D Printing Promises to Revolutionize Defense, Aerospace Industries," English, National Defense, vol. 98, no. 724, pp. 20-23, Mar. 2014.

[4] E. P. Flynn, "Low-cost approaches to UAV design using advanced manufacturing techniques," in 2013 IEEE Integrated STEM Education Conference (ISEC), 2013, pp. 1-4.

[5] P. Shakor, S. Nejadi, G. Paul, and S. Malek, "Review of Emerging Additive Manufacturing Technologies in 3D Printing of Cementitious Materials in the Construction Industry," Frontiers in Built Environment, vol. 4, no. January, 2019. 
[6] D. D. Camacho, P. Clayton, W. O’Brien, R. Ferron, M. Juenger, S. Salamone, and C. Seepersad, "Applications of Additive Manufacturing in the Construction Industry," in ISARC. Proceedings of the International Symposium on Automation and Robotics in Construction, vol. 34, Vilnius, 2017, pp. 1-8.

[7] W. Ostachowicz, R. Soman, and P. Malinowski, “Optimization of sensor placement for structural health monitoring: a review," Structural Health Monitoring, vol. 18, no. 3, pp. 963-988, 2019.

[8] G. Georgousis, C. Pandis, A. Kalamiotis, P. Georgiopoulos, A. Kyritsis, E. Kontou, P. Pissis, M. Micusik, K. Czanikova, J. Kulicek, and M. Omastova, "Strain sensing in polymer/carbon nanotube composites by electrical resistance measurement," Composites Part B: Engineering, vol. 68, pp. 162-169, 2015.

[9] M. I. N. P. Munasinghe, L. Miles, and G. Paul, "DirectWrite Fabrication of Wear Profiling IoT Sensor for 3D Printed Industrial Equipment," in International Symposium on Automation and Robotics in Construction, Banff, 2019, pp. 862-869.

[10] B. K. Mishra and A. Tripathy, "A preliminary study of particle separation in spiral concentrators using DEM," International Journal of Mineral Processing, vol. 94, no. 3-4, pp. 192-195, 2010.

[11] M. Dawoud, I. Taha, and S. J. Ebeid, "Strain sensing behaviour of 3D printed carbon black filled ABS," Journal of Manufacturing Processes, vol. 35, pp. 337-342, 2018.

[12] J. Zhao, K. Dai, C. Liu, G. Zheng, B. Wang, C. Liu, J. Chen, and C. Shen, "A comparison between strain sensing behaviors of carbon black/polypropylene and carbon nanotubes/polypropylene electrically conductive composites," Composites Part A: Applied Science and Manufacturing, vol. 48, no. 1, pp. 129-136, 2013.

[13] H. Nassar, M. Ntagios, W. T. Navaraj, and R. Dahiya, "Multi-material 3D Printed Bendable Smart Sensing Structures," in IEEE Sensors, 2018, pp. 1-4.

[14] C. Votzke, U. Daalkhaijav, Y. Mengue, and M. L. Johnston, "Highly-Stretchable Biomechanical Strain Sensor using Printed Liquid Metal Paste," in IEEE Biomedical Circuits and Systems Conference (BioCAS), Cleveland: IEEE, 2018, pp. 1-4.

[15] T. Yamada, Y. Hayamizu, Y. Yamamoto, Y. Yomogida, A. Izadi-Najafabadi, D. N. Futaba, and K. Hata, "A stretchable carbon nanotube strain sensor for humanmotion detection," Nature Nanotechnology, vol. 6, p. 296, Mar. 2011.

[16] C. Mattmann, F. Clemens, and G. Tröster, "Sensor for Measuring Strain in Textile," Sensors, vol. 8, no. 6, pp. 3719-3732, 2008.

[17] K. Elgeneidy, G. Neumann, M. Jackson, and N. Lohse, "Directly Printable Flexible Strain Sensors for Bending and Contact Feedback of Soft Actuators," Frontiers in Robotics and AI, vol. 5, no. 2, pp. 1-14, 2018.
[18] I. A. Anderson, T. A. Gisby, T. G. McKay, B. M. O'Brien, and E. P. Calius, "Multi-functional dielectric elastomer artificial muscles for soft and smart machines," Journal of Applied Physics, vol. 112, no. 4, 2012.

[19] A. Frutiger, J. T. Muth, D. M. Vogt, Y. Mengüç, A. Campo, A. D. Valentine, C. J. Walsh, and J. A. Lewis, "Capacitive soft strain sensors via multicoreshell fiber printing," Advanced Materials, vol. 27, no. 15, pp. 2440-2446, 2015.

[20] L. Pan, A. Chortos, G. Yu, Y. Wang, S. Isaacson, R. Allen, Y. Shi, R. Dauskardt, and Z. Bao, "An ultrasensitive resistive pressure sensor based on hollowsphere microstructure induced elasticity in conducting polymer film," Nature Communications, vol. 5, pp. 1-8, 2014.

[21] A. Nag, S. Feng, S. C. Mukhopadhyay, J. Kosel, and D. Inglis, "3D printed mould-based graphite/PDMS sensor for low-force applications," Sensors and Actuators, A: Physical, vol. 280, pp. 525-534, 2018.

[22] J. T. Muth, D. M. Vogt, R. L. Truby, D. B. Kolesky, R. J. Wood, and J. A. Lewis, "Embedded 3D Printing of Strain Sensors within Highly Stretchable Elastomers," Advanced Materials, vol. 26, no. 36, pp. 6307-6312, 2014.

[23] J. Chossat, Y. Park, R. J. Wood, and V. Duchaine, "A Soft Strain Sensor Based on Ionic and Metal Liquids," IEEE Sensors Journal, vol. 13, no. 9, pp. 3405-3414, 2013.

[24] National Instruments, "Measuring Strain with Strain Gauges," Tech. Rep., 2016. DOI: 10.1109/MCS.2009. 935588. [Online]. Available: http://www.ni.com/whitepaper/3642/en/.

[25] J. Rotheiser, Joining of Plastics: Handbook for Designers and Engineers, English. Munich; Cincinnati, OH: Hanser Publishers, 1999, p. 592, ISBN: 1569902534.

[26] LPKF Laser \& Electronics, "Laser Plastic Welding Design Guidelines Manual," Tech. Rep., pp. 0-30.

[27] M. J. Troughton, Handbook of plastics joining: a practical guide. William Andrew, 2008, ISBN: 0815519761.

[28] L. R. Lopes, A. F. Silva, and O. S. Carneiro, "Multimaterial 3D printing: The relevance of materials affinity on the boundary interface performance," Additive Manufacturing, vol. 23, pp. 45-52, 2018.

[29] K. R. Cheatle, Fundamentals of Test Measurement Instrumentation. 2006, pp. 263-269.

[30] A. International, ASTM D638-14, Standard Test Method for Tensile Properties of Plastics, West Conshohocken, PA, 2014. [Online]. Available: www.astm.org. 